\title{
RESISTENCIA A DROGAS DE SEGUNDA LIINEA EN CEPAS PERUANAS DE Mycobacterium tuberculosis MULTIDROGORRESISTENTES
}

\author{
Francesca Barletta1,2,a, Carlos Zamudio ${ }^{1,2, b}$, Leen Rigouts ${ }^{3, c}$, Carlos Seas $^{1,2, b}$
}

\section{RESUMEN}

Objetivos. Determinar los perfiles de resistencia de las quinolonas; ciprofloxacina (Cpx), ofloxacina (Ofx), gatifloxacina (Gfx) y moxifloxacina (Mfx), y de los inyectables; kanamicina $(\mathrm{Km})$, amikacina $(\mathrm{Am})$ y capreomicina $(\mathrm{Cm})$ en cepas multidrogorresistente (MDR). Se buscó la presencia de mutaciones en los genes rrs, tlyA y gyrA/B, y su posible asociación con la resistencia a inyectables y quinolonas. Materiales y métodos. En este estudio piloto descriptivo se seleccionaron cepas MDR aisladas durante junio a diciembre de 2004, que fueron criopreservadas en el banco de muestras del Instituto de Medicina Tropical "Alexander von Humboldt" en Lima, Perú. Se determinó la concentración mínima inhibitoria (CMI) para Cpx, Ofx, Gfx, Mfx, Km, Am y Cm. Se investigó las mutaciones presentes en los genes rrs, tlyA y gyrA/B a través de un PCR convencional y posterior secuenciamiento de los productos obtenidos. Resultados. Cuatro de los once aislados presentaron resistencia contra los inyectables y en todas se observó una alta $\mathrm{CMI} ;>120 \mu \mathrm{g} / \mathrm{mL}$ para Km $y>160 \mu \mathrm{g} / \mathrm{mL}$ para Am y Cm. Solo dos aislados presentaron resistencia a Ofx con un $\mathrm{CMI}=4 \mu \mathrm{g} / \mathrm{mL}$. Los resultados de secuenciamiento sugirieron que la mutación A1401T en rrs podría ser la causa molecular de resistencia a los inyectables; mientras que en este estudio no se halló ninguna mutación en tlyA ni en gyrA/B asociada a resistencia. Conclusiones. Este estudio sugiere una posible asociación entre la mutación en A1401G y la resistencia a los antibióticos inyectables.

Palabras clave: Resistencia a múltiples drogas; Mycobacterium tuberculosis; Farmacorresistencia microbiana (fuente: DeCS BIREME).

\section{RESISTANCE TO SECOND-LINE ANTI-TUBERCULOSIS DRUGS AMONG PERUVIAN MULTIDRUG RESISTANT Mycobacterium tuberculosis STRAINS}

\begin{abstract}
Objectives. To determine the drug resistance profiles for quinolones: ciprofloxacin (CFX), ofloxacin (OFX), moxifloxacin (MFX), and gatifloxacin (GFX); and for injectables: kanamycin (KAN), amikacin (AMK), and capreomycin (CAP) in multidrug resistant (MDR) strains. We also investigated the correlation between mutations in rrs, tlyA and gyrA/B genes, and the in vitro resistance to the second-line anti-tuberculosis drugs. Materials and methods. In this pilot study we selected MDR clinical isolates collected from June-December 2004 in the Tropical Medicine Institute "Alexander von Humboldt" (Lima, Perú). The Minimum Inhibitory Concentration (MIC) of CFX, OFX, MFX, GFX, KAN, AMK and CAP for 14 clinical isolates were determined and the sequences of rrs, tlyA and gyrA/B genes were analyzed by conventional PCR followed by sequencing. Results. We obtained valid results for 11 samples. Four isolates were resistant to injectable drugs, and in all the cases the MICs were; $>120 \mu \mathrm{g} / \mathrm{mL}$ for KAN and $>160 \mu \mathrm{g} / \mathrm{mL}$ for AMK and CAP. Only 2 isolates were resistant to OFX with MIC $=4 \mu \mathrm{g} / \mathrm{mL}$. Sequencing results suggested that the mutation A1401T in rrs gene could be the molecular cause of the resistance to injectable drugs. In this study we did not find any mutation in tlyA and gyrA/B associated to resistance. Conclusions. Our study suggests a possible association between the mutation A1401T in rrs and resistance to injectable drugs. However further studies should be done to confirm this hypothesis in Perú.
\end{abstract}

Key words: Drug resistance, multiple; Mycobacterium tuberculosis; Drug resistance, microbial (source: MeSH, NLM).

\footnotetext{
Instituto de Medicina Tropical "Alexander von Humboldt". Lima, Perú.

Universidad Peruana Cayetano Heredia. Lima, Perú.

Instituto de Medicina Tropical, University of Antwerp. Amberes, Bélgica.

Biólogo molecular; ${ }^{\mathrm{b}}$ Médico cirujano; ${ }^{\mathrm{c}}$ Biólogo microbiólogo

Recibido: : 21-04-14 Aprobado: 15-10-14
}

Citar como: Barletta F, Zamudio C, Rigouts L, Seas C. Resistencia a drogas de segunda línea en cepas peruanas de Mycobacterium tuberculosis multidrogorresistentes. Rev Peru Med Exp Salud Publica. 2014;31(4):676-82. 


\section{INTRODUCCIÓN}

El aumento de la tuberculosis con resistencia a los antibióticosisoniaziday rifampicina, omultidrogorresistente (TB-MDR) y la tuberculosis extensamente resistente (TBXDR) o resistente a isoniazida, rifampicina, y al menos a una fluoroquinolona y una droga antibiótica inyectable de segunda línea, amenaza los esfuerzos mundiales de control de la TB y es un problema de salud pública en varios países. En la región de las Américas, el Perú ocupa el cuarto lugar en incidencia de TB y el primer lugar en casos severos de TB-MDR ${ }^{(1)}$.

Aunque el Perú representa solo el $3 \%$ de la población de las Américas, el $12 \%$ de los pacientes con tuberculosis de la región y el $32 \%$ de los pacientes con TB-MDR residen en esta nación ${ }^{(2)}$. Dentro del país, las tasas de TB-MDR se distribuyen heterogéneamente. Las zonas urbanas son las más afectadas, pues $59 \%$ de todos los casos de TB y $82 \%$ de los casos de TB-MDR son reportados en Lima. Los casos de TB-XDR también se concentran en Lima $(93 \%)^{(3)}$. El primer caso de TB-XDR fue notificado en 1999 y hasta el 2012 se han registrado 434 casos de TB-XDR, de los cuales $89,4 \%$ se encontraban en Lima y la provincia del Callao ${ }^{(4)}$.

Las principales drogas bactericidas de segunda línea usadas en el tratamiento de TB pertenecen a los grupos de los inyectables y quinolonas. El primer grupo, conformado por aminoglicósidos y péptidos cíclicos, se conocen desde los 80 , pero eran aplicados para el tratamiento de otras enfermedades infecciosas. En Perú, los aminoglicósidos kanamicina $(\mathrm{Km})$ y amikacina $(\mathrm{Am})^{(5,6)}$, y el péptido capreomicina $(\mathrm{Cm})^{(7)}$ son usados actualmente en el tratamiento de TB-MDR. El grupo de las quinolonas se desarrolló entre las décadas de los 80 y 90 e incluyen compuestos de segunda, tercera y cuarta generación ${ }^{(8)}$. En Perú, la ciprofloxacin (Cpx) y ofloxacina (Ofx) han sido las quinolonas de elección hasta el $2013^{(9)}$. Sin embargo, actualmente el Programa Nacional de Tuberculosis recomienda el uso de levofloxacina (Lfx), el isómero levógiro de la Ofx ${ }^{(10,11)}$.

En 1991, con la implementación del DOTS (Directly Observed Therapy Short-course) en el Perú, se observó una disminución en la incidencia nacional de TB (1). Desafortunadamente, el aumento de casos de TBMDR y el acceso limitado a las pruebas de sensibilidad, dieron origen al tratamiento único (2RHZE/4R2H2) para todos los pacientes de TB; y posteriormente, en 1998, al tratamiento estandarizado para TB-MDR (2-4KmCxZEEtoCs/ Km2-3CxZEEto/ CxEtoCsZE*). Sin embargo, menos del $50 \%$ de los pacientes estaban curados al término del tratamiento y en aproximadamente el $30 \%$ se reportó falla al terapéutica ${ }^{(4)}$.
Frente a esta realidad, es necesario investigar la resistencia de cepas multidrogorresistentes a las drogas de segunda línea, no solo a nivel clínico sino a nivel molecular (12). El objetivo de este estudio piloto fue determinar el patrón de resistencia a los aminoglicósidos y las quinolonas en cepas de Mycobacterium tuberculosis multidrogorresistentes.

\section{MATERIALES Y MÉTODOS}

\section{POBLACIÓN}

El presente estudio descriptivo fue parte de un ensayo clínico (fase III), realizado en el periodo enero de 2004 a diciembre de 2006, en el que se compararon cuatro métodos para la determinación de resistencia de $M$. tuberculosis a drogas de primera línea. Para este estudio piloto se seleccionaron las cepas multidrogorresistente aisladas de pacientes durante junio a diciembre de 2004 que se encontraban criopreservadas en el Banco de muestras del Instituto de Medicina Tropical "Alexander von Humboldt" (Lima, Perú).

\section{CULTIVO}

Las cepas descriopreservadas fueron sembradas en 500 uL de caldo $7 \mathrm{H} 9$ suplementado con OADC (Oleic Albumin Dextrose Catalase) e incubadas a $37{ }^{\circ} \mathrm{C}$ durante dos semanas. Luego, se hizo un aislamiento en medio Löwenstein-Jensen y se incubó a $37{ }^{\circ} \mathrm{C}$ durante dos semanas. A partir de estos cultivos se prepararon soluciones bacterianas de $1 \mathrm{mg} / \mathrm{mL}$ y se sembraron los tubos para la determinación de la concentración mínima inhibitoria (Figura 1).

\section{DETERMINACIÓN DE LA CONCENTRACIÓN MÍNIMA INHIBITORIA (CMI)}

La determinación de $\mathrm{CMI}$ se realizó en medio Löwenstein-Jensen13; kanamicina ( $\mathrm{Km}: 7.5,15,30$, 60, $120 \mu \mathrm{g} / \mathrm{mL}$ ); amikacina (Am: 10, 20, 40, 80, $160 \mu \mathrm{g} /$ $\mathrm{mL})$; capreomicina $(\mathrm{Cm}$ : 10, 20, 40, 80, $160 \mu \mathrm{g} / \mathrm{mL})$; ciprofloxacina (Cpx: 1, 2, 4, $8 \mu \mathrm{g} / \mathrm{mL}$ ); ofloxacina (Ofx: $0.5,1,2,4,8 \mu \mathrm{g} / \mathrm{mL}$ ); gatifloxacina (Gfx: $0.25,0.5,1,2$, $4 \mu \mathrm{g} / \mathrm{mL}$ ), y moxifloxacina (Mfx: $0.5,1,2,4,8 \mu \mathrm{g} / \mathrm{mL}$ ) ${ }^{(14)}$.

Las drogas de segunda línea se obtuvieron de SigmaAldrich (de Bornem, Bélgica) o Acros Organon (Geel, Bélgica). Todos los tubos se incubaron a $37^{\circ} \mathrm{C}$ durante 28 días. La CMI se definió como la concentración más baja de fármaco que resulta en la inhibición del crecimiento $0<1 \%$ del inóculo. La concentración de corte de resistencia se definió de acuerdo con las recomendaciones de la Organización Mundial de la 


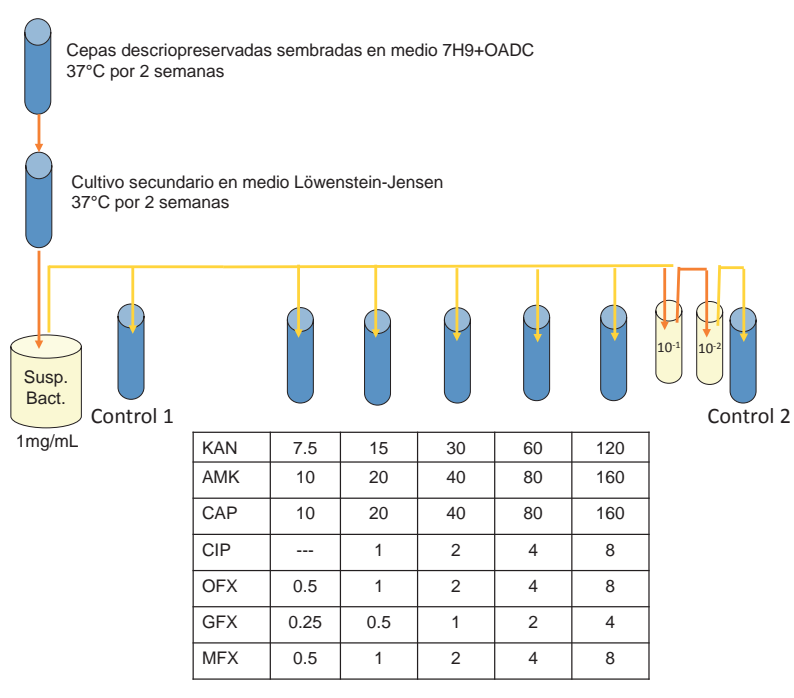

Figura 1. Determinación de la concentración mínima inhibitoria Las cepas descriopreservadas fueron sembradas en medio $7 \mathrm{H} 9+$ OADC. Luego se realizó un cultivo secundario en medio Löwenstein-Jenseny a partir de este se preparó un suspensión bacteriana de $1 \mathrm{mg} / \mathrm{mL}$. Se sembró un $1 \mathrm{~mL}$ en los tubos control y aquellos que contenían las diferentes concentraciones (ug/ $\mathrm{mL}$ ) de las drogas. Se preparó un segundo tubo control con una dilución $10^{-3}$ de la suspensión bacteriana inicial. La lectura se realizó a los 28 días de inoculación.

Salud (OMS) ${ }^{(15)}$ de la siguiente manera: $\mathrm{Km}-30 \mu \mathrm{g} / \mathrm{ml}$, $\mathrm{Am}-40 \mu \mathrm{g} / \mathrm{ml}, \mathrm{Cm}-40 \mu \mathrm{g} / \mathrm{ml}, \mathrm{Cpx}-2 \mu \mathrm{g} / \mathrm{mL}$, Ofx $-2 \mu \mathrm{g} /$ $\mathrm{mL}, \mathrm{Gfx}-1 \mu \mathrm{g} / \mathrm{mL}$ y Mfx $-2 \mu \mathrm{g} / \mathrm{mL}$.

\section{AISLAMIENTO DEL ADN}

Se tomaron colonias del tubo control con la ayuda de una asa de siembra de $10 \mathrm{uL}$ y se resuspedieron en $500 \mathrm{uL}$ de buffer TE 1X (Tris $10 \mathrm{mM}$, EDTA $1 \mathrm{mM}, \mathrm{pH}$ 8.0). Luego se incubaron a $95{ }^{\circ} \mathrm{C}$ durante 20 minutos para inactivar a las micobacterias. Finalmente, las muestras se centrifugaron a $13000 \mathrm{rpm}$ durante $15 \mathrm{~min}$ y los sobrenadantes fueron almacenados a $-20{ }^{\circ} \mathrm{C}$ para el posterior análisis de PCR.

\section{ENSAYO DE PCR Y SECUENCIAMIENTO}

Se usaron los siguientes sets de primers: KM-SA (5'-AAGTACCCCGCCTGGGGAG TACGG-3') y KM-RA (5'-GGTGGGACAACACCTGGAACAAGTC-3'), para la amplificación de un fragmento de 831 pb que contenía la región 3 'del gen rrs y parte de la región espaciadora adyacente (referida como "región 1400 rrs" a lo largo del texto); TlyA-SA(5'-CGACGTCGGTGGTGGTGCGGTA-3') y TIyA-RA (5'-GTCCGGTCTTCCACCCGGTAAT CCT-3), se usó para la amplificación de la región del gen tlyA de $810 \mathrm{pb}$; y GyraseB-sense
(5'-TAAGAGCGCCACCGACATCGGTGGATTG-3') y GyraseA-reverse (5'GATGAAA TCGACTGTCTCCTCGTCGATTTCCC-3') se usó para la amplificación del gen girasa de 1,231 bp (16,17).

El volumen final de reacción fue $50 \mu \mathrm{L}$ y contenía $10 \mathrm{mM}$ Tris- $\mathrm{HCl}(\mathrm{pH} 8,6), 50 \mathrm{mM} \mathrm{KCl}, 1,65 \mathrm{mM} \mathrm{MgCl} 2,200 \mu \mathrm{M}$ de cada uno de los cuatro deoxinucleótidos, $12,5 \mathrm{pmol}$ de cada primer, 1,5 U de enzima Taq polimerasa (Promega, Madison, WI) y $2 \mu \mathrm{L}$ del sobrenadante bacteriano inactivado. Se aplicaron las condiciones de PCR previamente descritas (Jugheli 2009) y se confirmó la presencia de productos en un gel de agarosa al $2 \%$.

Los productos fueron secuenciados en el Departamento de Genética de la Universidad de Amberes (Bélgica) en un secuenciador por capilaridad (Applied Biosystems 3730). Se usaron los primers KMSEQ (5'-CTAGAGATAGGCGTTCCCTT GTGG -3'), TlyA-SEQ (5'-CGATCGCACGTCGTCTTTCCGA-3') y GyraseABSeq (5'-GTCGATTTCCCTCAGCATCTCCATC-3') para secuenciar la hebra codante (contiene la información para la traducción de proteína) las regiones 1400 rrs, tlyA y gyrA/B, respectivamente. Las secuencias obtenidas fueron analizadas con el programa CLC Sequence Vewer (versión 7.0.1). Las mutaciones en rrs, tlyA y gyrA/B fueron identificadas con NCBI BLAST y fueron comparadas con la secuencia "wild type" $M$. tuberculosis H37Rv.

\section{CONSIDERACIONES ÉTICAS}

El ensayo clínico del que se desprendió el presente estudio piloto fue aprobado por el Comité de Ética de la Universidad Peruana Cayetano Heredia y del Instituto Nacional de Salud. Todos los pacientes enrolados firmaron un consentimiento informado y los datos se gestionaron de manera anónima.

\section{RESULTADOS}

Durante junio a diciembre de 2004 se procesaron 444 muestras de esputos provenientes de 444 pacientes que presentaron TB pulmonar diagnosticada por microscopia. Del total de muestras analizadas, $27(6,1 \%)$ cepas aisladas fueron resistentes a isoniazida $(\mathrm{H})$ y rifampicina $(\mathrm{R})$, pero solo $18(66,7 \%)$ fueron criopreservadas. Al descriopreservar y sembrar los cultivos secundarios se obtuvieron $14(77,8 \%)$ cultivos positivos y 4 $(22,2 \%)$ cultivos negativos. Finalmente, se realizó la determinación de la CMI a drogas de segunda línea en 14 cepas aisladas de pacientes que no habían recibido previamente tratamiento para TB-MDR (Figura 2). 


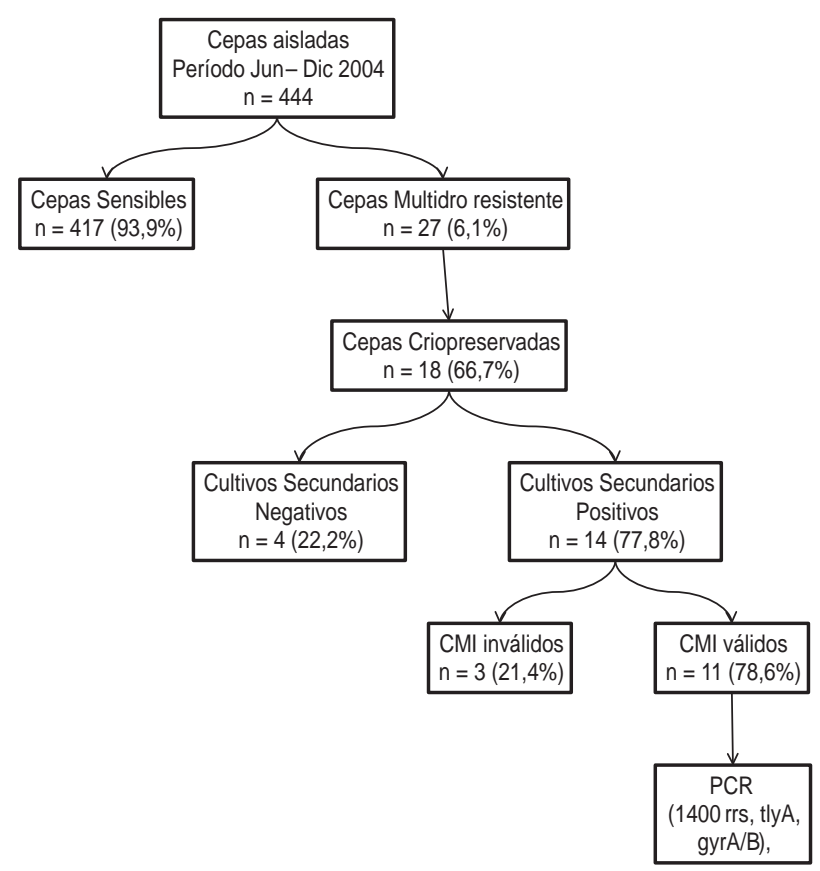

Figura 2. Flujograma: cepas multidrogorresistentes

\section{DETERMINACIÓN DE LA CONCENTRACIÓN MÍNIMA INHIBITORIA (CMI)}

Del total de analizados, $11(78,6 \%)$ cepas sembradas para la determinación de la $\mathrm{CMI}$ tuvieron resultados válidos mientras que $3(21,4 \%)$ cepas no presentaron crecimiento en los tubos controles invalidando los resultados. Cuatro $(36,4 \%)$ cepas presentaron resistencia a todos los aminoglicósidos y la CMI fue $>120 \mu \mathrm{g} / \mathrm{mL}$ para $\mathrm{Km}$ y $>160 \mu \mathrm{g} / \mathrm{mL}$ para Am y Cm. Las siete $(63,6 \%$ ) cepas restantes fueron susceptibles a todos los aminoglicósidos y la CMl estuvo en un rango de 7,5 a $30 \mu \mathrm{g} / \mathrm{mL}$ para $\mathrm{Km}$ y en un rango de 10 a $40 \mu \mathrm{g} / \mathrm{mL}$ para Am y $\mathrm{Cm}$. En el caso de las quinolonas se observó un rango de $\mathrm{CMI}$ bajo para todas las drogas (Gfx y Mfx, 0,25 $\mu \mathrm{g} / \mathrm{mL}$; Cx, $1-2$ $\mu \mathrm{g} / \mathrm{mL}$; Ofx, $0.5-2 \mu \mathrm{g} / \mathrm{mL}$ ) y solo dos cepas presentaron resistencia intermedia a Ofx $(\mathrm{CMI}=4 \mu \mathrm{g} / \mathrm{mL})$.

\section{ASOCIACIÓN ENTRE LA RESISTENCIA IN VITRO Y MUTACIONES EN rrs, tlyA Y gyrA/B}

Se secuenciaron en total 33 productos de PCR pertenecientes a 11 aislados diferentes y se observaron dos mutaciones en el gen tlyA (A33G, A750T), una mutación en el gen rrs (A1401G), 1 mutación en gyrA (T95(ACC)) y 1 mutación en gyrB (T695(ACC)). Las mutaciones A33G y A750T estuvieron presentes en todas las cepas $(n=11)$, sin embargo, no se observó asociación con la resistencia a aminoglicósidos. La mutación A1401G presente en el gen rrs se observó en $4 / 11(36,4 \%)$ aislados y todas mostraron resistencia a los tres aminoglicósidos con $\mathrm{CMI}>120 \mu \mathrm{g} / \mathrm{mL}$ para $\mathrm{Km}$ y $>160 \mu \mathrm{g} / \mathrm{mL}$ para Am y Cm. La mutación T695 (ACC) se observó en una sola cepa resistente, mientras que la mutación T95 (ACC) se observó en 5/11 cepas (Tabla 1).

Tabla 1. Mutaciones en los genes $r r s$, tlyA y gyrA/B; CMI determinadas por la prueba de proporciones en medio Löwenstein-Jensen y perfiles de susceptibilidad de los 11 aislados de $M$. tuberculosis

\begin{tabular}{|c|c|c|c|c|c|c|c|c|c|c|c|c|c|c|c|c|c|}
\hline \multirow[b]{2}{*}{ ID } & \multicolumn{3}{|c|}{ Mutaciones en: } & \multicolumn{7}{|c|}{ CMI (mg/L) } & \multicolumn{7}{|c|}{ Patrones de susceptibilidad } \\
\hline & rrs (nctd) & tlyA (nctd) & $\begin{array}{c}g y r A / B \\
\text { (codon) }\end{array}$ & $\mathrm{Km}$ & Am & $\mathrm{Cm}$ & Cx & Ofx & Gfx & Mfx & $\mathrm{Km}$ & Am & $\mathrm{Cm}$ & Cx & Ofx & Gfx & Mfx \\
\hline 1 & & $\begin{array}{l}\text { A33G } \\
\text { A750T }\end{array}$ & & 15 & 20 & 20 & 2 & 2 & 0.25 & 0.5 & S & S & $\mathrm{S}$ & $S$ & $S$ & S & S \\
\hline 2 & & $\begin{array}{l}\text { A33G } \\
\text { A750T }\end{array}$ & & 15 & 20 & 20 & 1 & 2 & 0.25 & 0.5 & $S$ & $S$ & S & $S$ & S & S & S \\
\hline 3 & A1401G & $\begin{array}{l}\text { A33G } \\
\text { A750T }\end{array}$ & T95(ACC) & $>120$ & $>160$ & $>160$ & 2 & 2 & 0.25 & 0.25 & $\mathrm{R}$ & $\mathrm{R}$ & $\mathrm{R}$ & $S$ & $S$ & $S$ & $S$ \\
\hline 4 & & $\begin{array}{l}\text { A33G } \\
\text { A750T }\end{array}$ & & 7.5 & 10 & 10 & 1 & 2 & 0.25 & 0.25 & $S$ & $S$ & $S$ & $S$ & $S$ & $S$ & $S$ \\
\hline 5 & & $\begin{array}{l}\text { A33G } \\
\text { A750T }\end{array}$ & $\begin{array}{l}\text { T95(ACC) } \\
\text { T695(ACC) }\end{array}$ & 7.5 & 20 & 10 & 1 & 2 & 0.25 & 0.5 & $S$ & $S$ & $S$ & $S$ & $S$ & $S$ & $S$ \\
\hline 6 & & $\begin{array}{l}\text { A33G } \\
\text { A750T }\end{array}$ & & 15 & 10 & 10 & 2 & 2 & 0.25 & 0.25 & $S$ & $S$ & $S$ & $S$ & $S$ & $S$ & $S$ \\
\hline 7 & A1401G & $\begin{array}{l}\text { A33G } \\
\text { A750T }\end{array}$ & T95(ACC) & $>120$ & $>160$ & 160 & 2 & 2 & 0.25 & 0.25 & $\mathrm{R}$ & $\mathrm{R}$ & $\mathrm{R}$ & $S$ & $S$ & $S$ & $S$ \\
\hline 8 & A1401G & $\begin{array}{l}\text { A33G } \\
\text { A750T }\end{array}$ & T95(ACC) & $>120$ & $>160$ & $>160$ & 2 & 4 & 0.25 & 0.5 & $\mathrm{R}$ & $\mathrm{R}$ & $\mathrm{R}$ & $S$ & $\mathrm{R}$ & $S$ & $S$ \\
\hline 9 & A1401G & $\begin{array}{l}\text { A33G } \\
\text { A7850 }\end{array}$ & T95(ACC) & $>120$ & $>160$ & 160 & 2 & 4 & 0.25 & 0.5 & $\mathrm{R}$ & $\mathrm{R}$ & $\mathrm{R}$ & $S$ & $\mathrm{R}$ & $S$ & $S$ \\
\hline 10 & & $\begin{array}{l}\text { A33G } \\
\text { A750T }\end{array}$ & & 30 & 10 & 20 & 2 & 2 & 0.25 & 0.25 & $S$ & $S$ & $S$ & S & $S$ & $S$ & $S$ \\
\hline 11 & & $\begin{array}{l}\text { A33G } \\
\text { A750 }\end{array}$ & & 30 & 20 & 10 & 1 & 0.5 & 0.25 & 1 & $S$ & $S$ & $S$ & $S$ & $S$ & $\underline{\mathrm{S}}$ & $S$ \\
\hline
\end{tabular}

Abreviaturas: $\mathrm{Km}=$ kanamicina, $\mathrm{Am}=a m i k a c i n a, \mathrm{Cm}=$ capreomicina, $\mathrm{Cpx}=$ ciprofloxacina, Ofx=ofloxacina, $\mathrm{G} \mathrm{f}=$ gatifloxacina, Mfx=moxifloxacina, $M A=$ mutaciones ausentes, $R=$ resistente, $S=$ sensible, nctd=nucleótido 


\section{DISCUSIÓN}

En el presente estudio piloto se observó una baja resistencia a quinolonas, a pesar que la ciprofloxacina es la droga de elección para una variedad de infecciones en Perú. Sin embargo, la presencia de resistencia a inyectables genera preocupación debido a los altos valores de $\mathrm{CMI}$ que se observaron, sugiriendo que estos ya no serían adecuados para el tratamiento de casos de TB-MDR.

Las quinolonas son una familia de antibióticos de gran importancia en el manejo de muchas enfermedades comunitarias y nosocomiales. Debido al uso inadecuado de las quinolonas, han comenzado a presentarse resistencia de diversos gérmenes a estas ${ }^{(18)}$. En la actualidad existe una gran variedad de fluoroquinolonas, las más usadas son Cpx, Ofx, Lfx, Mfx y Gfx.

A pesar que en el Perú se usan con frecuencia las quinolonas contra un amplio espectro de enfermedades infecciosas, en nuestro estudio piloto no se observó resistencia. En el Perú, la Cpx es la droga de elección para una variedad de infecciones, entre las cuales están la TB pulmonar ${ }^{(19)}$. Estudios in vitro han demostrado que la Cpx tiene una baja actividad bactericida temprana contra $M$. tuberculosis y que se requeriría una dosis diaria mínima de $1000 \mathrm{mg}$, la cual es superior a la CMI de la Cpx $(0,25-2 \mu \mathrm{g} / \mathrm{mL})^{(20,21)}$. Sin embargo, la baja resistencia que se observó podría deberse a que la micobacteria, al ser fagocitada por el macrófago, se mantendría protegida en el fagosoma, mientras que la Cpx se acumularía en el citoplasma de la célula ${ }^{(22,23)}$. Además, Seral et al. también postularon la existencia de una bomba de flujo que eliminaría a la $\mathrm{Cpx}$ del citoplasma de las células reduciendo aun más las posibilidades de interacción entre la droga y la micobacteria.

Por otro lado, los inyectables son prescritos para el tratamiento de infecciones urinarias altas complicadas (pielonefritis aguda), sepsis (en combinación con otros antibióticos), endocarditis por Streptococcus viridans (junto con penicilina), entre otras infecciones. Tulkens et al. demostraron que los inyectables son fácilmente incorporados en fagocitos cuando las células están en contacto con estos durante períodos prolongados permitiendo que las micobacterias estén expuestas a ellos. Esto explicaría la resistencia observada en algunos aislados en este estudio piloto ${ }^{(24)}$.

El mecanismo molecular para la mayoría de las drogas de segunda línea usadas en TB es conocido ${ }^{(25)}$. La resistencia a inyectables como $\mathrm{Am}, \mathrm{Km}$ y $\mathrm{Cm}$ estaría asociada a mutaciones en el gen rrs de la región $16 S$ rRNA, específicamente entre los nucleótidos 1400-
$1500{ }^{26)}$. En el caso de la capreomicina se postula además que la resistencia también podría estar asociada a mutaciones en el gen tlyA que codifica la 2-O-metiltransferesa ${ }^{(27)}$. Por su parte, las quinolonas tienen como blanco la enzima topoisomerasa II (ADN girasa) codificada por los genes gyrA y gyrB. Mutaciones en dos regiones de estos genes (A90, D94, A74, G88, S91, N533), conocidas como regiones determinantes de resistencia a quinolonas, estarían asociadas a la resistencia a las principales quinolonas ${ }^{(17,28)}$.

Con el fin de entender el mecanismo molecular de la resistencia, se deben descartar las mutaciones que no confieren resistencia a los inyectables y quinolonas. En este estudio piloto, las mutaciones A33G y A750T en el gen tlyA estuvieron presentes en todos los aislados (resistentes y sensibles), y no presentaron asociación con resistencia a ningún aminoglucósido. Así mismo, las mutaciones presentes en los genes gyrA y gyrB asociadas a los codones T95 y T695, no presentaron asociación con resistencia a ninguna quinolona.

Estudios previos han demostrado que la mutación G1484T en el gen rrs está asociada con la resistencia a $\mathrm{Km}, \mathrm{Am}$ y $\mathrm{Cm} 29$. Además, Maus et al. demostraron que algunas mutaciones en el gen tlyA conferían resistencia a $\mathrm{Cm}$. En el presente estudio ninguno de los aislados analizados presentó alguna de estas mutaciones. Sin embargo, las mutaciones previamente reportadas en el gen tlyA fueron encontradas en mutantes generadas en el laboratorio pero en ninguno de los aislados clínicos analizados ${ }^{(27)}$

La correlación entre la mutación A1401T en el gen rrs y la resistencia a los tres inyectables que se observó en nuestro estudio fue más clara y fuerte en comparación a los últimos datos reportados ${ }^{(27,29)}$. Aunque este resultado puede estar sesgado por el amplio uso indebido de $\mathrm{Km}$ y Am en el Perú, es posible que aun represente una realidad en todo el mundo, y por lo tanto la mutación A1401G podría ser la causa principal de resistencia clínicamente significativa in vivo. Además, el método molecular GenoType MTBDRsl (Hain Lifescience, Nehren, Alemania) (30), actualmente utilizado en el Instituto Nacional de Salud, contiene la mutación A1401T en su panel de detección (11).

El presente estudio presentó las siguientes limitaciones: la selección de cepas MDR, el período relativamente corto de siete meses de recolección de muestras, y la falta de representatividad del número de muestras.

Se concluye que los resultados obtenidos sugieren una baja resistencia a quinolonas y la posibilidad de continuar su uso de manera segura para el tratamiento de TB- 
XDR. Además, sugiere una posible asociación entre la mutación en A1401G y la resistencia a inyectables en cepas MDR. Recomendamos realizar estudios futuros abarcando un período mayor e incluir cepas sensibles y multidrogorresistentes para poder dilucidar las mutaciones que realmente estarían asociadas con la resistencia a inyectables.

Agradecimientos: A nuestras trabajadoras de campo por la recolección de datos y muestras; y al personal de los centros de salud por su apoyo continúo en la conducción del estudio.
Fuentes de financiamiento: Este estudio fue financiado por la Dirección General de Cooperación Belga para el Desarrollo (DGDC) a través de una colaboración institucional entre el Instituto de Medicina Tropical de Amberes, Bélgica y el Instituto de Medicina Tropical Alexander von Humboldt en Lima, Perú.

Conflictos de interés: Los autores declaran no tener conflictos de interés.

\section{REFERENCIAS BIBLIOGRÁFICAS}

1. World Health Organization. Global Tuberculosis Report 2012. Geneva: WHO; 2012.

2. World Health Organization. Multidrug and extensively drug-resistant TB (M/ XDR-TB):

2010 global report on surveillance and response. Geneva: WHO; 2010.

3. Bonilla C. Situación de la tuberculosis en el Perú. Acta Med Per 2008 JulSet;25(3):163-70.

4. Ministerio de Salud. Impacto socioeconómico de la tuberculosis en el Perú 2010: Documento técnico. Lima: MINSA; 2012.

5. Pestka S. The use of inhibitors in studies on protein synthesis. Methods Enzymol. 1974;30:261-82.

6. Kawaguchi H. Discovery, chemistry, and activity of amikacin. J Infect Dis. 1976 Nov;134 SUPPL:S242-8.

7. Stark WM, Higgins CE, Wolfe RN, Hoehn MM, McGuire JM. Capreomycin, a new antimycobacterial agent produced by Streptomyces capfeolu J sp. n. Antimicrobial Agents and Chemotherapy. 1962:596-606.

8. Norris S, Mandell GL. The quinolones: history and overview. San Diego: Academic Press Inc. 1988.

9. Mendoza-Ticona A, Moore DA, Alarcón V, Samalvides F, Seas C. Propuesta de esquemas de tratamiento antituberculosis basados en la susceptibilidad A isoniacida y rifampicina. Rev Peru Med Exp Salud Publica. 2013; 30(2):197-204.

10. Morrissey I, Hoshino K, Sato K, Yoshida A, Hayakawa I, Bures MG, et al. Mechanism of differential activities of ofloxacin enantiomers. Antimicrob Agents Chemother. 1996 Aug;40(8):1775-84.
11. Perú, Ministerio de Salud. Aprueban "Norma Técnica de Salud para la atención integral de las personas afectadas por tuberculosis. Resolución Ministerial No 715. Aprobada el 8 de Noviembre del 2013. El Peruano (14 noviembre del 2013).

12. Dalton T, Cegielski P, Akksilp S, Asencios L, Campos Caoili J, Cho $\mathrm{SN}$, et al. Prevalence of and risk factors for resistance to second-line drugs in people with multidrug-resistant tuberculosis in eight countries: a prospective cohort study. Lancet. 2012 Oct 20;380(9851):1406-17. doi: 10.1016/S0140-6736(12)60734-X.

13. Canetti G, Rist N, Grosset J. Mesure de la sensibilité du bacille tuberculeux aux drugues antibacillaires par la méthode des proportions. Rev Tuber 1963;27:217-72.

14. Kam KM, Sloutsky A, Yip CW, Bulled N, Seung KJ, Zignol M, et al. Determination of critical concentrations of second-line antituberculosis drugs with clinical and microbiological relevance. Int J Tuberc Lung Dis. 2010;14(3):282-8.

15. World Health Organization. Policy guidance on drug-susceptibility testing (DST) of second-line antituberculosis drugs. WHO/HTM/TB/2008.392. Geneva: WHO; 2008.

16. Jugheli L, Bzekalava N, de Rijk P, Fissette K, Portaels F, Rigouts L. High level of crossresistance between kanamycin, amikacin, and capreomycin among Mycobacterium tuberculosis isolates from Georgia and a close relation with mutations in the rrs gene. Antimicrob Agents Chemother. 2009 Dec;53(12):5064-8. doi: 10.1128/ AAC.00851-09.
17. Von Groll A, Martin A, Jureen P, Hoffner S, Vandamme P, Portaels $\mathrm{F}$, et al. Fluoroquinolone resistance in Mycobacterium tuberculosis and mutations in gyrA and gyrB. Antimicrob Agents Chemother. 2009 Oct;53(10):4498-500. doi: 10.1128/ AAC.00287-09.

18. Hooper DC. New uses for new and older quinolones and the challenge of resistance. Clin Infect Dis. 2000 Feb;30(2):243-54.

19. Maguiña C, Solari L. Nuevas y viejas quinolonas. Rev Med Hered. 2002 Oct-Dic;13(4):153-60.

20. Kennedy N, Fox R, Kisyombe GM, Saruni AO, Uiso LO, Ramsay AR, et al. Early bactericidal and sterilizing activities of ciprofloxacin in pulmonary tuberculosis. Am Rev Respir Dis. 1993 Dec;148(6 Pt 1):1547-51.

21. Kennedy N, Berger L, Curram J, Fox R, Gutmann J, Kisyombe GM, et al. Randomized controlled trial of a drug regimen that includes ciprofloxacin for the treatment of pulmonary tuberculosis. Clin Infect Dis. 1996 May;22(5):827-33.

22. Carryn S, Van Bambeke F, MingeotLeclercq MP, Tulkens PM. Comparative intracellular (THP-1 macrophage) and extracellular activities of betalactams, azithromycin, gentamicin, and fluoroquinolones against Listeria monocytogenes at clinically relevant concentrations. Antimicrob Agents Chemother. 2002 Jul;46(7):2095-103.

23. Seral C, Van Bambeke F, Tulkens PM. Quantitative analysis of gentamicin, azithromycin, telithromycin, ciprofloxacin, moxifloxacin, and oritavancin (LY333328) activities against intracellular Staphylococcus 
aureus in mouse J774 macrophages. Antimicrob Agents Chemother. 2003 Jul;47(7):2283-92.

24. Tulkens P, Trouet A. Uptake and intracellular localization of kanamycin and gentamycin in the lysosomes of cultured fibroblasts. Arch Int Physiol Biochim. 1974 Dec;82(5):1018-9.

25. Almeida Da Silva PE, Palomino JC. Molecular basis and mechanisms of drug resistance in Mycobacterium tuberculosis: classical and new drugs. J Antimicrob Chemother. 2011 Jul;66(7):1417-30. doi: $10.1093 / \mathrm{jac} / \mathrm{dkr} 173$.

26. Alangaden GJ, Kreiswirth BN, Aouad A, Khetarpal M, Igno FR, Moghazeh SL, et al. Mechanism of resistance to amikacin and kanamycin in Mycobacterium tuberculosis. Antimicrob. Agents Chemother. 1998 May;42(5):1295-7.
27. Maus CE, Plikaytis BB, Shinnick TM. Mutation of tlyA confers capreomycin resistance in Mycobacterium tuberculosis. Antimicrob. Agents Chemother. 2005 Feb;49(2):571-7.

28. Pitaksajjakul P, Wongwit W, Punprasit W, Eampokalap B, Peacock S, Ramasoota P. Mutations in the gyrA and gyrB genes of fluoroquinoloneresistant Mycobacterium tuberculosis from TB patients in Thailand. Southeast Asian J Trop Med Public Health. 2005;36 Suppl 4:228-37.

29. Suzuki Y, Katsukawa C, Tamaru A, Abe C, Makino M, Mizuguchi Y, et al. Detection of kanamycin-resistant Mycobacterium tuberculosis by identifying mutations in the $16 \mathrm{~S}$ rRNA gene. J. Clin. Microbiol. 1998 May;36(5):1220-5.

30. Brossier F, Veziris N, Aubry A, Jarlier V, Sougakoff W. Detection by GenoType
MTBDRsl test of complex mechanisms of resistance to second-line drugs and ethambutol in multidrug-resistant Mycobacterium tuberculosis complex isolates. J Clin Microbiol. 2010 May;48(5):1683-9. doi: 10.1128/ JCM.01947-09.

Correspondencia: Francesca Barletta Solari Dirección: Universidad Peruana Cayetano Heredia. Av. Honorio Delgado No340, San Martin de Porres.

Teléfono: 4823903 anexo 20

Correo electrónico:francescabarletta@yahoo.es

\section{Consulte la versión electrónica de la} Revista Peruana de Medicina Experimental y Salud Pública en www.pubmed.gov

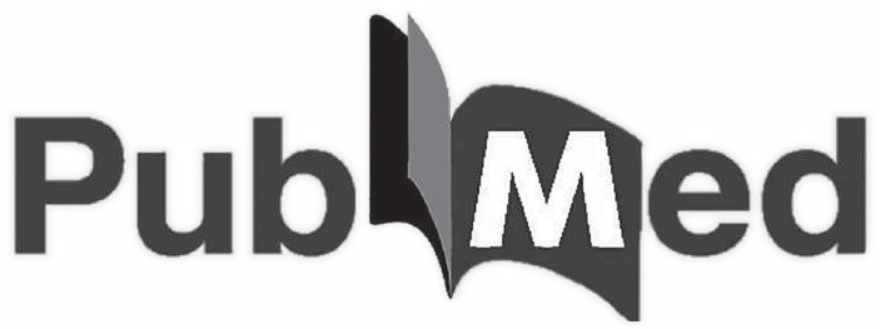

